\title{
A Hybrid Material of Graphene and Poly (3,4-ethyldioxythiophene) with High Conductivity, Flexibility, and Transparency
}

\author{
Yanfei Xu, Yan Wang, Jiajie Liang, Yi Huang, Yanfeng Ma, Xiangjian Wan, and Yongsheng Chen（ $\varangle)$
}

Key Laboratory for Functional Polymer Materials and Centre for Nanoscale Science and Technology, Institute of Polymer Chemistry, College of Chemistry, Nankai University, Tianjin 300071, China

Received: 24 January 2009/Revised: 18 February 2009/Accepted: 18 February 2009

(O)Tsinghua University Press and Springer-Verlag 2009. This article is published with open access at Springerlink.com

\begin{abstract}
A novel hybrid material prepared from graphene and poly (3,4-ethyldioxythiophene) (PEDOT) shows excellent transparency, electrical conductivity, and good flexibility, together with high thermal stability and is easily processed in both water and organic solvents. Conductivities of the order of $0.2 \mathrm{~S} / \mathrm{cm}$ and light transmittance of greater than $80 \%$ in the $400-1800 \mathrm{~nm}$ wavelength range were observed for films with thickness of tens of $\mathrm{nm}$. Practical applications in a variety of optoelectronic devices are thus expected for this transparent and flexible conducting graphene-based hybrid material.
\end{abstract}

\section{KEYWORDS}

Graphene-poly (3,4-ethyldioxythiophene) (PEDOT) optical transparency, conductivity, flexibility, stability

In recent years, transparent and flexible conducting materials have pervaded modern technologies, providing a critical component of touch screens, video displays, and plastic solar cells [1-3]. Traditionally, Sn-doped $\operatorname{In}_{2} \mathrm{O}_{3}$ (ITO) deposited on flexible substrates has been used to meet the demand for such materials. However, ITO is relatively brittle, so that cracks appear after repeated bending or strain, and furthermore it is not resistant to acid and indium compounds are generally rather toxic. In addition, ITO is becoming increasingly expensive due to the limited availability of the element indium. In respond to these technical problems, an extensive search is being carried out for alternative materials which are environmentally friendly and stable, with high conductivity and good transparency as well as the ability to be processed in solution.

Organic conducting polymers have received increasing attention as possible transparent and conductive materials because of their efficacious optical [4] and electronic [5] properties, and potential applications in flexible electronic devices [6, 7]. Poly (3,4-ethyldioxythiophene) (PEDOT) represents one such conjugated polymer that can be used as the active material in flexible organic electronics due to its remarkably high conductivity, transparency, and environmental stability [8], but its insolubility appears to be a major drawback in terms of processing for incorporation in devices. This solubility problem can be overcome by using a water-soluble polyelectrolyte poly (styrenesulfonic acid) (PSS), as a chargebalancing dopant in hybrids such as PSS/PEDOT

Address correspondence to yschen99@nankai.edu.cn 
[8]. Obviously, water is not a particularly good processing solvent for organic optoelectronic devices. Furthermore, PEDOT/PSS often suffers from low conductivity. As such, much research has been devoted to find a simple organic solution-based route to fabricate highly transparent electrically conductive thin films.

Considering the conductivity dependence of the doped PEDOT on its counteranions, one method to enhance its conductivity is to exploit new chargebalancing dopants [9]. Graphene, a new class of two-dimensional $\mathrm{sp}^{2}$ carbon nanostructure, holds great promise for potential applications in many technological fields such as nanoelectronics [10, 11]. Thus, it is to be expected that novel hybrid materials with high conductivity, transparency, and flexibility will result if graphene-based materials are used in an appropriate form as the doping material for PEDOT.

Herein, we report a novel hybrid material (graphene-PEDOT) with good organic solutionprocessablity, excellent optical transparency, conductivity, and flexibility. The structure and synthesis of the graphene-PEDOT hybrid are represented schematically in Schemes 1 and 2. Briefly, the monomer 3,4-ethyldioxythiophene (EDOT) was polymerized in situ [12] in a solution of sulfonated graphene to give graphene-PEDOT.

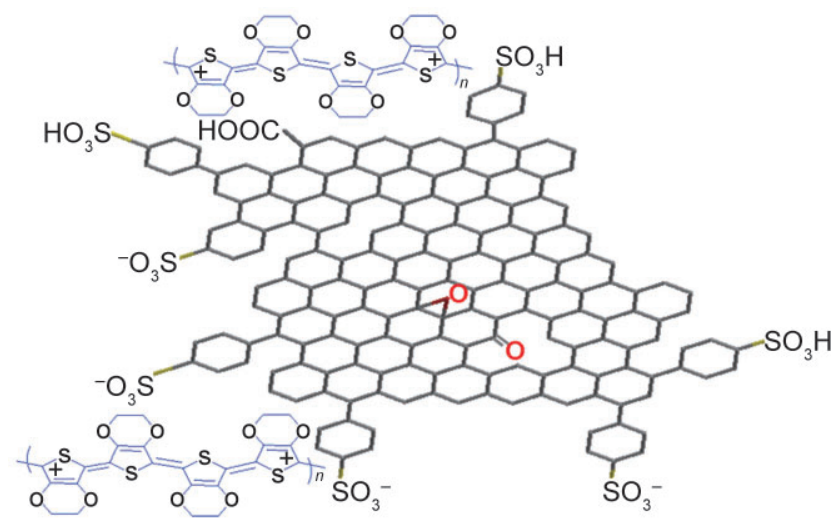

Scheme 1 Schematic representation of part of the structure of graphene-PEDOT

$$
\begin{aligned}
& \begin{array}{l}
\text { Sulfonated-graphene } \\
+\mathrm{EDOT} \\
+\mathrm{Fe}_{2}\left(\mathrm{SO}_{4}\right)_{3}
\end{array} \frac{\text { in situ }}{\text { polymerization }} \text { Graphene-PEDOT }
\end{aligned}
$$

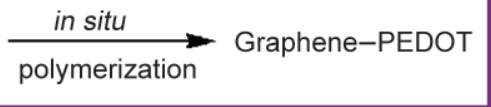

Scheme 2 Synthesis of graphene-PEDOT. Reagents and conditions: Sulfonated graphene $(10 \mathrm{mg})$, anhydrous $\mathrm{Fe}_{2}\left(\mathrm{SO}_{4}\right)_{3}(10 \mathrm{mg}, 0.025$ $\mathrm{mmol})$, EDOT (100 mg, $0.70 \mathrm{mmol}), \mathrm{H}_{2} \mathrm{O}(10 \mathrm{~mL}), 50{ }^{\circ} \mathrm{C}, 48 \mathrm{~h}$
Perfect graphene does not exist naturally, but bulk and aqueous/organic solution-processable functionalized graphene materials can now be prepared easily [13-19]. However, the presence of foreign stabilizers is undesirable for most applications [20]. New strategies to produce relatively clean graphene sheets in bulk quantity while keeping them individually separated are required. Furthermore, an appropriate doping group for PEDOT needs to be incorporated in graphene. Based on these considerations, we have developed a two-step method for the preparation of a sulfonated graphene material, which shows good solubility in both water and organic solvents $(12 \mathrm{mg} / \mathrm{mL})$. Using a modified procedure reported by Samulski [21], we first prepared a lightly sulfonated graphene, but with an improved water solubility $(2 \mathrm{mg} / \mathrm{mL})$. This was further functionalized with arylsulfonic acid in fuming $\mathrm{H}_{2} \mathrm{SO}_{4}$ to obtain our sulfonated graphene with additional sulfonate groups [22]. (See Electronic Supplementary Material (ESM) for details of the synthesis of sulfonated graphene and graphenePEDOT.) Atomic force microscopy (AFM) (Fig. S-1 in the ESM) confirms that this further functionalized graphene material can be easily dissolved, almost entirely as individual graphene sheets, in water. AFM was also used to probe graphene-PEDOT dispersion in solvents, film morphology, and thickness (Fig. S-2 in the ESM).

In the Fourier transform in frared (FTIR) spectra of highly sulfonated graphene (Fig. 1), the peaks at 1187, 1123, and $1037 \mathrm{~cm}^{-1}$ (two $v_{\mathrm{S}-\mathrm{O}}$ and one $v_{\mathrm{S}-\text { phenyl }}$ bands) confirm the presence of the sulfonic acid group [21]. The successful formation of the PEDOT in graphene-PEDOT is evidenced by the presence of the peaks at 1522 and $1316 \mathrm{~cm}^{-1}(\mathrm{C}=\mathrm{C}$ and $\mathrm{C}$ -C stretching vibrations of the thiophene ring), 1199 and $1087 \mathrm{~cm}^{-1}$ (C-O-C bond stretching), and 982, 839, and $689 \mathrm{~cm}^{-1}$ (C-S bond in the thiophene ring) [23]. Furthermore, a peak at $1513 \mathrm{~cm}^{-1}$ corresponding to the $\mathrm{C}=\mathrm{C}$ stretching vibration of the quinoidal structure originating from the thiophene ring was clearly observed, indicating that the PEDOT is doped by sulfonated graphene as in the case of PSS-PEDOT [24].

The prevention of aggregation is of particular 


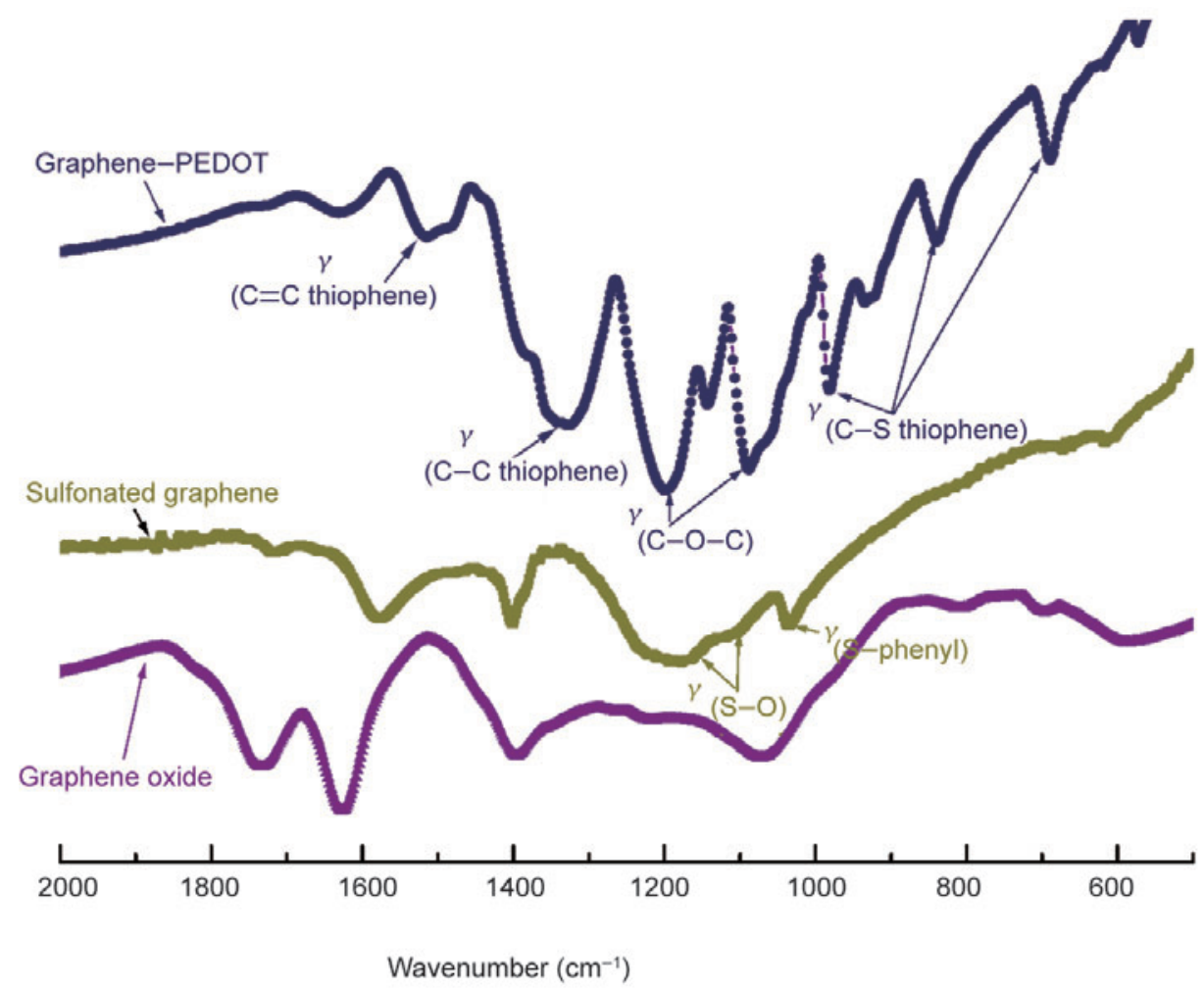

Figure 1 FTIR spectra of graphene oxide, sulfonated graphene and graphene-PEDOT. The band at 1513 $\mathrm{cm}^{-1}$ in the spectrum of graphene-PEDOT, arising from the $C=C$ stretching vibration of the quinoidal structure originating from the thiophene ring, indicates the successful formation of the doped PEDOT

importance for processability and applications of graphene because most of its attractive properties are only associated with individual graphene sheets. Solution-phase UV-Vis-NIR spectroscopy (Fig. 2) can be used to determine the solubility of graphene -PEDOT [25]. The absorptions (at $267 \mathrm{~nm}$ ) for the graphene moiety [15] in the hybrid were plotted against concentration (Fig. 2 inset) and a good linear relationship was obtained with an $R$ value of 0.9996 . Assuming the applicability of Beer's law, from the slope of the linear least-squares fit we estimated the effective extinction coefficient of the graphene -PEDOT to be $0.014 \mathrm{~L} \cdot \mathrm{mg}^{-1} \cdot \mathrm{cm}^{-1}$ at this position. The absorbance of solutions of graphene-PEDOT at other wavelengths was also consistent with the Beer's law. Similar results were obtained for the UV -Vis-NIR spectra of graphene-PEDOT in DMF (Fig. S-3 in the ESM). These results demonstrate that graphene-PEDOT has good solubility in both water and DMF.

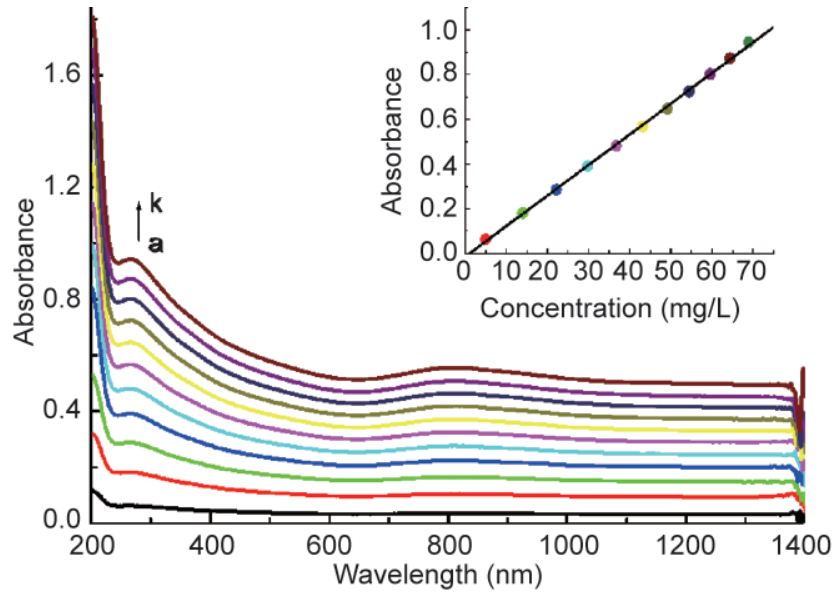

Figure 2 Concentration dependence of UV absorption of graphenePEDOT in $\mathrm{H}_{2} \mathrm{O}$ (concentrations are 4.9, 14.0, 22.2, 29.8, 36.7, 43.1, $49.1,54.5,80.2,87.3,94.5 \mathrm{mg} / \mathrm{L}$, from a to $\mathrm{k}$, respectively). Shown in the inset is a plot of optical density at maximal absorption position (267 $\mathrm{nm})$ for the graphene moiety versus concentration. The straight line in the plot in the inset is a linear least-squares fit to the data, indicating the hybrid graphene-PEDOT was dissolved homogeneously in the solvent 


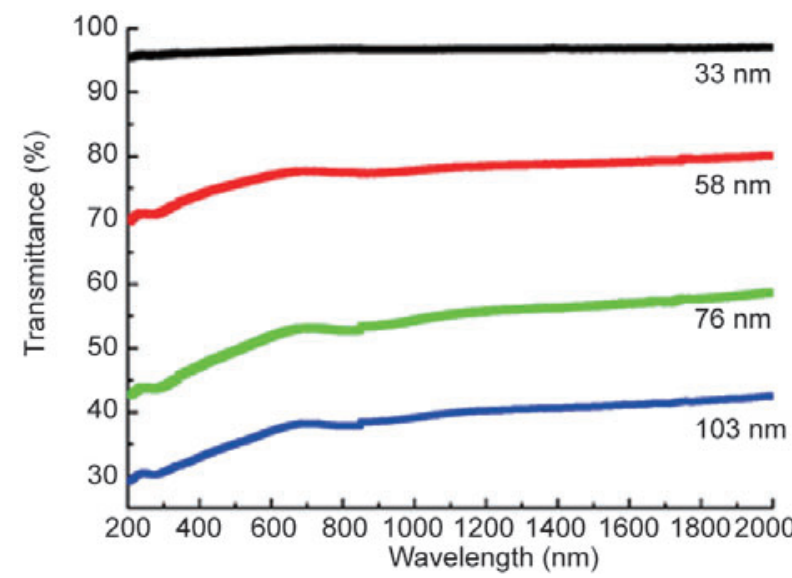

(a)

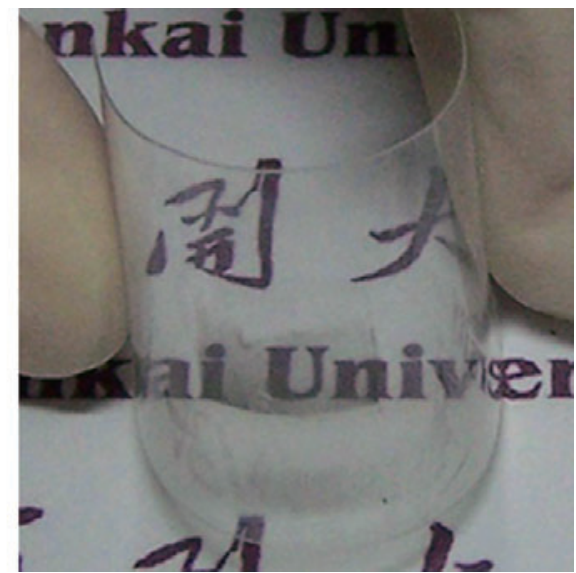

(b)

Figure 3 (a) Optical transmittance spectra of films with different thicknesses on a quartz substrate; (b) a picture of a graphene-PEDOT film with a thickness of $\sim 32 \mathrm{~nm}$ on a transparent PMMA substrate

Next, we investigated the transparency of the graphene-PEDOT films with various thicknesses on a quartz substrate (Fig. 3 (a)). The thickness of the graphene-PEDOT film on quartz and its transparency could be tuned by varying the solution concentration. More importantly, the graphenePEDOT films are essentially highly transparent over the whole 200-2000 nm wavelength range. GraphenePEDOT films with thickness of 33, 58, 76, and 103 nm have transmittances of $96 \%, 76 \%, 51 \%$, and $36 \%$, respectively, at a wavelength of $550 \mathrm{~nm}$.

Films of this hybrid with different thicknesses show excellent conductivity, and more importantly retain their high conductivity when even bent by over $90^{\circ}$. Films were also prepared by drop-casting aqueous / DMF solutions of graphene-PEDOT onto different substrates. The films are relatively uniform and their conductivity is almost independent of the film thickness. For example, films with a thickness of 35 to $119 \mathrm{~nm}$ prepared from aqueous solution on a quartz substrate all showed a conductivity of $\sim 0.2 \mathrm{~S} / \mathrm{cm}$. A 40-nm-thick film prepared from DMF solution on a quartz substrate showed a conductivity of $0.07 \mathrm{~S} / \mathrm{cm}$, whilst a $172-\mathrm{nm}$-thick film prepared from DMF solution on a polymethyl methacrylate (PMMA) substrate had a conductivity of $0.108 \mathrm{~S} / \mathrm{cm}$. More importantly, when the film on the PMMA was bent (Fig. $3($ b)), its high conductivity was retained fairly well. For example, after the above 172-nmthick PMMA film was bent inwards, its conductivity became $0.18 \mathrm{~S} / \mathrm{cm}$; while when the film was bent outwards, its conductivity became $1.3 \times 10^{-4} \mathrm{~S} / \mathrm{cm}$. Finally, when the bending force was released and the film became flat again, its conductivity returned to about the same value $(0.104 \mathrm{~S} / \mathrm{cm})$. Furthermore, no observable cracks were observed on the graphenePEDOT film. This new flexible graphene hybrid has significantly higher conductivity than that of a commercial PEDOT-PSS product (Clevios ${ }^{\mathrm{TM}} \mathrm{P}$ AI $4083,10^{-5}-10^{-6} \mathrm{~S} / \mathrm{cm}$ ). The conductivity is sufficient for many applications of transparent conducting materials [20]. Furthermore, the conductivity can be expected to be improved through a more complete

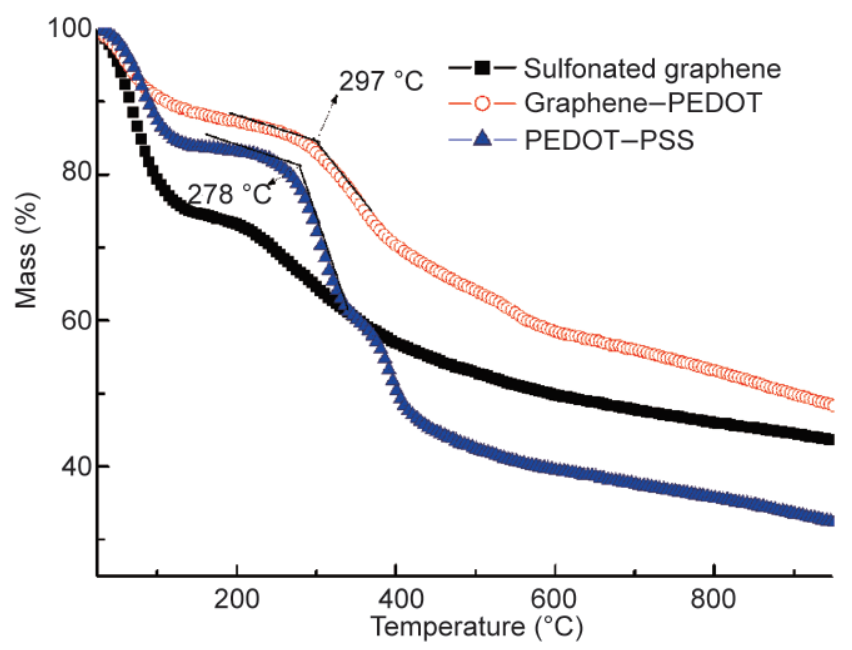

Figure 4 TGA spectra of sulfonated graphene, graphene-PEDOT and PSS-PEDOT obtained with a heating rate of $5^{\circ} \mathrm{C} / \mathrm{min}$ from room temperature to $400{ }^{\circ} \mathrm{C}$ under $\mathrm{N}_{2}$ 
reduction/annealing [26].

The thermal stability of a hybrid is critical as far as potential applications are concerned and the thermal stability of hybrid Graphene-PEDOT was therefore investigated by thermogravimetric analysis (TGA). The TGA results of graphene -PEDOT, PSS-PEDOT (Clevios ${ }^{\mathrm{TM}}$ P AI 4083) together with that for sulfonated graphene are shown in Fig. 4. All three samples show a slight mass loss above $100{ }^{\circ} \mathrm{C}$, which can be attributed to the loss of adsorbed solvent [18]. Graphene -PEDOT shows excellent thermal stability with its second onset degradation temperature above $297{ }^{\circ} \mathrm{C}$ and only a very small mass loss below this temperature. The total mass loss below $325{ }^{\circ} \mathrm{C}$ was $\sim 19 \%$. As for PSS-PEDOT, the second significant mass loss occurred at $278{ }^{\circ} \mathrm{C}$, accounted for by decomposition of PSS-PEDOT and this decomposition temperature is $18{ }^{\circ} \mathrm{C}$ lower than that $\left(297^{\circ} \mathrm{C}\right)$ of graphene-PEDOT. There is a total mass loss of $\sim 35 \%$ below $325{ }^{\circ} \mathrm{C}$ for PSS-PEDOT, significantly higher than that for graphene-PEDOT. These results demonstrate that graphene-PEDOT has better thermal stability than the commercial PSS-PEDOT.

In summary, a novel graphene hybrid material showing both water and organic processability has been prepared. Thin films of the material show excellent transparency, electrical conductivity, and stability. Significantly, this material is robust and flexible, while retaining high electrical conductivity after deformation. These outstanding properties, combined with the ease of its preparation and solution processing capability, give this graphenePEDOT material great potential for application in various devices where a combination of conductivity, transparency and flexibility are demanded.

\section{Acknowledgements}

The authors gratefully acknowledge the financial support from the Natural Science Foundation of china (NSFC) (\#20774047), the Ministry of Science and Techology of china (MoST) (\#2006CB932702), and natural Science Foundation (NSF) of Tianjin City (\#07JCYBJC03000, \#08JCZDJC25300).
Electronic Supplementary Material: Supplementary material is available in the online version of this article at http://dx.doi.org/10.1007/s12274-009-9032-9 and is accessible free of charge.

\section{References}

[1] Eda, G.; Fanchini, G.; Chhowalla, M. Large-area ultrathin films of reduced graphene oxide as a transparent and flexible electronic material. Nat. Nanotechnol. 2008, 3, 270-274.

[2] Watcharotone, S.; Dikin, D. A.; Stankovich, S.; Piner, R.; Jung, I.; Dommett, G. H. B.; Evmenenko, G.; Wu, S. E.; Chen, S. F.; Liu, C. P.; Nguyen, S. T.; Ruoff, R. S. Graphene-silica composite thin films as transparent conductors. Nano Lett. 2007, 7, 1888-1892.

[3] Wang, X.; Zhi, L. J.; Tsao, N.; Tomovic, Z.; Li, J. L.; Müllen, $\mathrm{K}$. Transparent carbon films as electrodes in organic solar cells. Angew. Chem, Int. Ed. 2008, 47, 2990-2992.

[4] Patil, A. O.; Heeger, A. J.; Wudl, F. Optical properties of conducting polymers. Chem. Rev. 1988, 88, 183-200.

[5] Frommer, J. E. Conducting polymer solutions. Acc. Chem. Res. 1986, 19, 2-9.

[6] Heeger, A. J. Semiconducting and metallic polymers: The fourth generation of polymeric materials (Nobel Lecture). Angew. Chem. Int. Ed. 2001, 40, 2591-2611.

[7] MacDiarmid, A. G. Synthetic metals: A novel role for organic polymers (Nobel Lecture). Angew. Chem. Int. Ed. 2001, 40, 2581-2590.

[8] Groenendaal, B. L.; Jonas, F.; Freitag, D.; Pielartzik, H.; Reynolds, J. R. Poly (3,4-ethylenedioxythiophene) and its derivatives: Past, present, and future. Adv. Mater. 2000, 12, 481-494.

[9] Fan, B. H.; Mei, X. G.; Ouyang, J. Y. Significant conductivity enhancement of conductive poly (3,4-ethylenedioxythiophene) : Poly (styrenesulfonate) films by adding anionic surfactants into polymer solution. Macromolecules 2008, 41, 5971-5973.

[10] Novoselov, K. S.; Geim, A. K.; Morozov, S. V.; Jiang, D.; Zhang, Y.; Dubonos, S. V.; Grigorieva, I. V.; Firsov, A. A. Electric field effect in atomically thin carbon films. Science 2004, 306, 666-669.

[11] Liu, Z.; Liu, Q.; Huang, Y.; Ma, Y.; Yin, S.; Zhang, X.; Sun, W.; Chen, Y. Organic photovoltaic devices based on a novel acceptor material: Graphene. Adv. Mater. 2008 20, 3924-3930 
[12] Jonas, F.; Krafft, W. Eur. Patent 440957 to Bayer AG, 1991.

[13] McAllister, M. J.; Li, J. L.; Adamson, D. H.; Schniepp, H. C.; Abdala, A. A.; Liu, J.; Herrera-Alonso, M.; Milius, D. L.; Caro, R.; Prud'homme, R. K.; Aksay, I. A. Single sheet functionalized graphene by oxidation and thermal expansion of graphite. Chem. Mater. 2007, 19, 43964404.

[14] Ramanathan, T.; Abdala, A. A.; Stankovich, S.; Dikin, D. A.; Herrera-Alonso, M.; Piner, R. D.; Adamson, D. H.; Schniepp, H. C.; Chen, X.; Ruoff, R. S.; Nguyen, S. T.; Aksay, I. A.; Prud'Homme, R. K.; Brinson, L. $C$. Functionalized graphene sheets for polymer nanocomposites. Nat. Nanotechnol. 2008, 3, 327-331.

[15] Li, D.; Müller, M. B.; Gilje, S.; Kaner, R. B.; Wallace, G. G. Processable aqueous dispersions of graphene nanosheets. Nat. Nanotechnol. 2008, 3, 101-105.

[16] Niyogi, S.; Bekyarova, E.; Itkis, M. E.; McWilliams, J. L.; Hamon, M. A.; Haddon, R. C. Solution properties of graphite and graphene. J. Am. Chem. Soc. 2006, 128, 7720-7721.

[17] Stankovich, S.; Piner, R. D.; Nguyen, S. T.; Ruoff, R. S. Synthesis and exfoliation of isocyanate-treated graphene oxide nanoplatelets. Carbon 2006, 44, 3342-3347.

[18] Park, S.; An, J.; Piner, R. D.; Jung, I.; Yang, D.; Velamakanni, A.; Nguyen, S. T.; Ruoff, R. S. Aqueous suspension and characterization of chemically modified graphene sheets. Chem. Mater. 2008, 20, 6592-6594.

[19] Fan, X.; Peng, W.; Li, Y.; Li, X.; Wang, S.; Zhang, G.; Zhang, F. Deoxygenation of exfoliated graphite oxide under alkaline conditions: A green route to graphene preparation. Adv. Mater. 2008, 20, 4490-4493.

[20] Stankovich, S.; Dikin, D. A.; Dommett, G. H. B.; Kohlhaas, K. M.; Zimney, E. J.; Stach, E. A.; Piner, R. D.; Nguyen, S. T.; Ruoff, R. S. Graphene-based composite materials. Nature 2006, 442, 282-286.

[21] Si, Y.; Samulski, E. T. Synthesis of water soluble graphene. Nano Lett. 2008, 8, 1679-1682.

[22] Yi, B.; Rajagopalan, R.; Foley, H. C.; Kim, U. J.; Liu, X. M.; Eklund, P. C. Catalytic polymerization and facile grafting of poly (furfuryl alcohol) to single-wall carbon nanotube: preparation of nanocomposite carbon. J. Am. Chem. Soc. 2006, 128, 11307-11313.

[23] Han, M. G.; Foulger, S. H. 1-Dimensional structures of poly (3,4-ethylenedioxythiophene) (PEDOT) : A chemical route to tubes, rods, thimbles, and belts. Chem. Commun. 2005, 3092-3094.

[24] Kvarnstrom, C.; Neugebauer, H.; Ivaska, A.; Sariciftci, N. S. Vibrational signatures of electrochemical $p$ - and n-doping of poly (3,4-ethylenedioxythiophene) films: An in situ attenuated total reflection Fourier transform infrared (ATR-FTIR) study. J. Mol. Struct. 2000, 521, 271277.

[25] Guo, Z.; Du, F.; Ren, D.; Chen, Y.; Zheng, J.; Liu, Z.; Tian, J. Covalently porphyrin-functionalized single-walled carbon nanotubes: A novel photoactive and optical limiting donor-acceptor nanohybrid. J. Mater. Chem. 2006, 16, 3021-3030.

[26] Becerril, H. A.; Mao, J.; Liu, Z.; Stoltenberg, R. M.; Bao, Z.; Chen, Y. Evaluation of solution-processed reduced graphene oxide films as transparent conductors. ACS Nano 2008, 2, 463-470. 